\title{
ZONEAMENTO BIOCLIMÁTICO PARA VACAS LEITEIRAS NO ESTADO DA BAHIA
}

\author{
SÍLVIA H. N. TURCO², THIERES G. F. DA SILVA ${ }^{3}$, LUIZ F. C. DOS SANTOS ${ }^{3}$, \\ PAULO H. B. RIBEIRO ${ }^{4}$, GHERMAN G. L. DE ARAÚJO ${ }^{5}$, \\ EVANDRO V. HOLANDA JUNIOR ${ }^{5}$, MÔNICA A. AGUIAR ${ }^{6}$
}

\begin{abstract}
RESUMO: Foram utilizados dados meteorológicos médios diários de 437 estações meteorológicas distribuídas por todo o Estado da Bahia para realizar o zoneamento bioclimático de vacas leiteiras e o declínio da produção de leite para vacas com nível de produção de 10 e $25 \mathrm{~kg} \mathrm{dia}^{-1}$, nas condições térmicas desse Estado. As variáveis consideradas foram a temperatura (Ta) e a umidade relativa do ar (UR) que foram utilizadas no cálculo do índice de temperatura e umidade (ITU). Estimou-se o declínio da produção de leite (DPL) em função do ITU e do nível de produção (NP). Com os valores de ITU e DPL, foram traçadas as isolinhas dessas duas variáveis para a Bahia por meio do programa SURFER 7.0. Observou-se que, mesmo para os meses com temperaturas mais amenas, há grande possibilidade de ocorrência de estresse climático para vacas em lactação, em algumas regiões do Estado. Nos meses mais quentes, há três zonas bioclimáticas na Bahia com maior ocorrência de estresse. Com a espacialização do declínio na produção, foram verificadas zonas com maior probabilidade de ocorrência de decínio na produção de leite, devido às condições climáticas desfavoráveis, apresentando regiões com perdas de até $1 \mathrm{~kg}$ de leite por vaca por dia com NP de $10 \mathrm{~kg}$ e regiões com perdas de produção de até $4,5 \mathrm{~kg}$ de leite por vaca por dia com NP de $25 \mathrm{~kg}$.
\end{abstract}

PALAVRAS-CHAVE: produção de leite, índice de temperatura e umidade, conforto térmico.

\section{BIOCLIMATICAL ZONING OF MILK COWS IN THE STATE OF BAHIA, BRAZIL}

ABSTRACT: Daily mean meteorological data from 437 meteorological stations in the State of Bahia were used to accomplish the bioclimatical zoning of milk cows and the decline in milk yield of cows with production levels of 10 and $25 \mathrm{~kg} \mathrm{day}^{-1}$ in summer thermic condition in this State. The variables temperature (Ta) and relative air humidity $(\mathrm{RH})$ were used to calculate the temperature and humidity index (THI). The milk production decline (MPD) was estimated based on data of the THI and the level of production (LP). With these data the PMD and THI mapping was done for the State of Bahia with the program SURFER 7.0. It can be observed that through the zoning, in despite the lowest temperatures in cold months, a bioclimatical area shows great possibilities for climatical stress of milk cows. During the hottest months there are three bioclimatical zones in the State of Bahia with a great occurrence of climatical stress. With the maps, areas with great probabilities for decline in milk production were observed due to unfavorable weather conditions. Those areas show production losses up to $1 \mathrm{~kg}$ for cows with (NP) of $10 \mathrm{~kg}$ and zones with losses as much as $4.5 \mathrm{~kg}$ for cows with (NP) of $25 \mathrm{~kg}$.

KEYWORDS: milk yield, temperature and humidity index, thermic comfort.

\footnotetext{
${ }^{1}$ Trabalho financiado pelo Programa PROAP/UNEB.

${ }^{2}$ Eng ${ }^{a}$ Agrícola, Professora Adjunta, Departamento de Tecnologia e Ciências Sociais, UNEB, Juazeiro - BA, Fone: (0XX74) 611.7363, silviaturco@aol.com

${ }^{3}$ Graduando de Agronomia, Departamento de Tecnologia e Ciências Sociais, UNEB, Bolsista do PROFIC/UNEB, Juazeiro - BA.

${ }^{4}$ Gerente Regional do Setor de Recursos Hídricos, SRH, Juazeiro - BA.

${ }_{6}^{5}$ Pesquisador da Embrapa Semi-Árido, Petrolina - PE.

${ }^{6}$ Professora Adjunta da UFSC.

Recebido pelo Conselho Editorial em: 20-11-2004

Aprovado pelo Conselho Editorial em: 13-4-2006
} 


\section{INTRODUÇÃO}

Altas temperaturas do ar, principalmente quando associadas a altas umidades e à radiação solar direta, são os principais elementos climáticos estressores causadores de baixo desempenho do gado leiteiro. As vacas leiteiras de raças especializadas em lactação e de média e alta produção são particularmente sensíveis ao estresse por calor, devido à função produtiva especializada e à alta eficiência na utilização dos alimentos, como conseqüência da intensa produção de calor associada à digestibilidade e ao metabolismo de grandes quantidades de nutrientes. A redução na produção de leite das vacas sob estresse térmico deve-se, primordialmente, ao menor consumo de alimentos, à hipofunção da tireóide e ao gasto de energia necessária para dissipação de calor corporal (BACCARI JÚNIOR, 1998).

Vários índices foram desenvolvidos com o objetivo de expressar o conforto do animal em relação ao ambiente. $\mathrm{O}$ índice mais utilizado é o de temperatura e umidade (ITU), que combina os efeitos da temperatura e da umidade relativa sobre o desempenho de bovinos, originalmente desenvolvido por THON (1958). Bovinos de todas as idades mostraram graus mensuráveis de desconforto térmico com o ITU igual ou superior a 78, e o desconforto tornou-se agudo à medida que o índice aumentou (McDOWELL \& JOHNSTON , 1971). JOHNSON (1980) observou que, para valores de ITU a partir de 72, a produção de leite foi declinando, sendo o declínio mais acentuado nas vacas de mais altas produções.

Em termos de produção de leite, IGONO et al. (1992) determinaram para vacas holandesas, na região central do Arizona (clima quente e seco), os valores críticos mínimo, médio e máximo de ITU de 64; 72 e 76, respectivamente, encontrando redução na produção de 11,5 a 16,0 kg por vaca, por dia, quando comparada às produções de verão com as de meses com temperaturas mais amenas no Arizona. OLIVEIRA (1980), citado por CAMPOS et al. (2002), argumenta que, para valores de ITU de 70 ou menos, vacas leiteiras apresentam quase nenhum desconforto térmico. Entretanto, para valores de 75 ou mais, a produção de leite e a ingestão de alimentos são seriamente prejudicados.

Este trabalho teve o objetivo de estabelecer o zoneamento bioclimático e a espacialização do declínio da produção de leite das vacas leiteiras da raça holandesa com nível de produção de 10 e $25 \mathrm{~kg}$ por dia, para condições de verão e de inverno, no Estado da Bahia, que possuem ITU elevados e considerados estressantes para a exploração do gado leiteiro, resultando em declínio acentuado na produção.

\section{MATERIAL E MÉTODOS}

Utilizaram-se os dados meteorológicos médios diários dos últimos 30 anos, de 437 estações meteorológicas distribuídas por todo o Estado da Bahia. As variáveis consideradas foram: temperatura (Ta) e a umidade relativa do ar (UR). Nas estações meteorológicas que não possuíam dados de UR, foram utilizados os valores do índice hídrico para estimar a UR. Tais valores foram obtidos a partir da equação de regressão desenvolvida para a Bahia por TEIXEIRA et al. (2001).

Depois de estimada a UR, essa e a Ta foram utilizadas para determinar os valores do índice de temperatura e umidade para os meses mais quentes e frios de cada estação meteorológica, segundo BUFFINGTON et al. (1982):

$$
\mathrm{ITU}=0,8 \mathrm{Ta}+\mathrm{UR}(\mathrm{Ta}-14,3) / 100+46,3
$$

em que,

Ta - temperatura do bulbo seco, ${ }^{\circ} \mathrm{C}, \mathrm{e}$

UR - umidade relativa do ar, \%. 
Já para estimar as perdas de produção de leite decorrentes dos fatores climáticos na Bahia, foi utilizada a equação proposta por HAHN (1993), ou seja: em que,

$$
\mathrm{DPL}=-1,075-1,736(\mathrm{NP})+0,02474(\mathrm{NP})(\mathrm{ITU})
$$

DPL - declínio absoluto na produção de leite, kg por vaca por dia;

NP - nível normal de produção de leite, kg por vaca por dia, e

ITU - valor médio diário do índice de temperatura e umidade (calculado para o zoneamento bioclimático), adimensional.

O declínio na produção de leite foi estimado em função de dois níveis normais de produção por vaca (NP): 10 e $25 \mathrm{~kg}$ por vaca, por dia. O NP representa o potencial de produção dos animais em condições de conforto térmico, ou seja, em condições de termoneutralidade.

Com os dados do ITU e DPL, juntamente com os dados geográficos de longitude, latitude e altitude de cada estação meteorológica, foi possível espacializar o ITU e o DPL em pontos de grade.

Os pontos foram calculados para intervalos de meio grau de latitude e longitude, cobrindo toda a área do Estado da Bahia, nos quais foram traçadas as isolinhas com essa disposição para os meses mais quentes e mais frios de cada estação meteorológica, dos dados do ITU e do DPL, para os dois níveis de produção de 10 e $25 \mathrm{~kg}$ por vaca, por dia. Os mapas foram traçados por meio do software SURFER 7.0 .

\section{RESULTADOS E DISCUSSÃO}

Na Figura 1, observa-se o Zoneamento Bioclimático para bovinos leiteiros da Bahia, baseado no ITU para os meses mais frios do ano. A Zona Bioclimática I apresentou ITU na faixa de 73 a 75 , sendo considerado alerta para vacas holandesas, de acordo com o NATIONAL WEATHER SERVICE (1976), citado por BAETA \& SOUZA (1997), os quais publicaram que valores para o índice de temperatura e umidade de 74 a 78 exigem cuidado, alerta.

A Zona Bioclimática I também se encontra em estado de alerta, conforme a classificação de ROSENBERG et al. (1983), que classificam o ITU na seguinte amplitude: entre 75 e 78 significa alerta para os produtores e providências são necessárias para evitar perdas.

Nenhuma das zonas bioclimáticas esteve dentro das faixas de perigo ou extremo perigo, respectivamente, de 79 a 83, e maior ou igual a 84, situações em que são necessárias medidas de segurança que devem ser empreendidas para evitar perdas desastrosas (ROSENBERG et al., 1983).

A Zona Bioclimática I localiza-se na Região Semi-Árida Baiana, onde ocorrem altas temperaturas e baixa umidade do ar. O zoneamento pode auxiliar nas decisões de ações que minimizem o estresse calórico, tais como sombreamento para os animais não-confinados nos horários mais quentes do dia que, segundo COLLIER et al. (1981), podem reduzir a freqüência respiratória e aumentar a produção de leite. ARCARO JÚNIOR et al. (2001) também compararam vacas em lactação em três tipos de instalação: sombra, sombra mais ventilação forçada e sombra com ventilação forçada associada à aspersão. Os autores relatam freqüência respiratória mais baixa dos animais na instalação com ventilação forçada e produção de leite mais alta para os animais submetidos à sombra com ventilação associada à aspersão. 


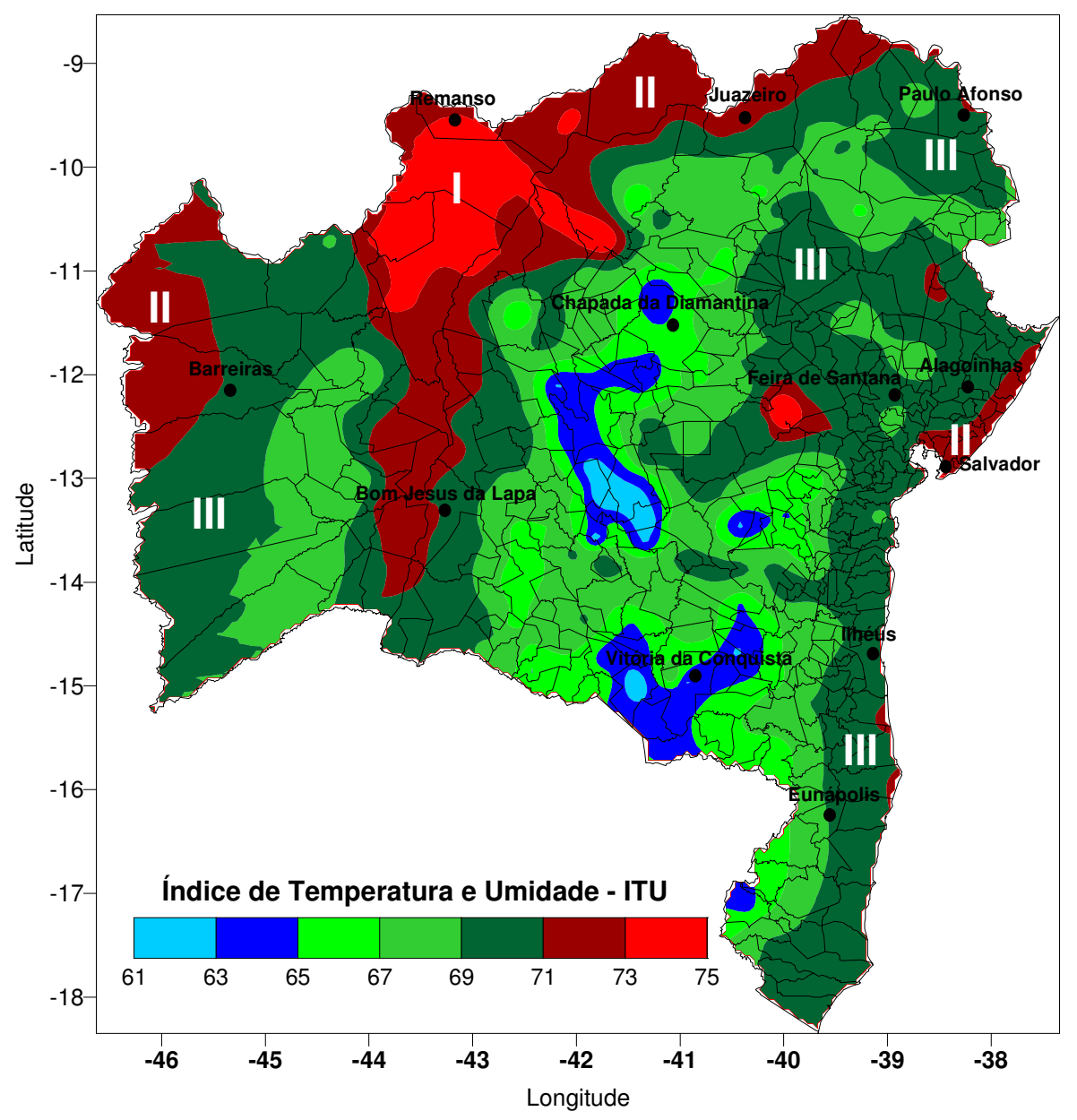

FIGURA 1. Zoneamento Bioclimático para bovinos, baseado no índice de temperatura e umidade (ITU) referente aos meses mais frios do ano para a Bahia.

Na Figura 2, observa-se o Zoneamento Bioclimático para bovinos leiteiros da Bahia, baseado no ITU para os meses mais quentes do ano. Observam-se as Zonas Bioclimáticas II e III, que apresentam valores de ITU de 73 a 77, considerado ambiente estressante para os animais e de cuidado ou alerta, e a Zona Bioclimática I apresenta valores de ITU maiores que 77, considerados de perigo para as vacas leiteiras (ROSENBERG et al., 1983). Nessas condições ambientais termicamente desconfortáveis, as vacas holandesas tendem a reduzir consideravelmente o consumo de matéria seca (MS), na tentativa de diminuir a taxa metabólica e a conseqüente produção de calor metabólico (COLLIER \& BEEDE, 1985). Vários autores indicam que a redução no consumo voluntário de alimentos tem sido a principal razão dos decréscimos na produção de leite em vacas submetidas ao estresse pelo calor (CHEN et al., 1993; McDOWELL et al., 1976). A região Central, onde se localiza a Chapada da Diamantina, e o Centro-Sul do Estado, onde se localiza a cidade de Vitória da Conquista, mesmo nas condições climáticas de verão, apresentaram ITU considerados dentro das condições de conforto para vacas holandesas. 


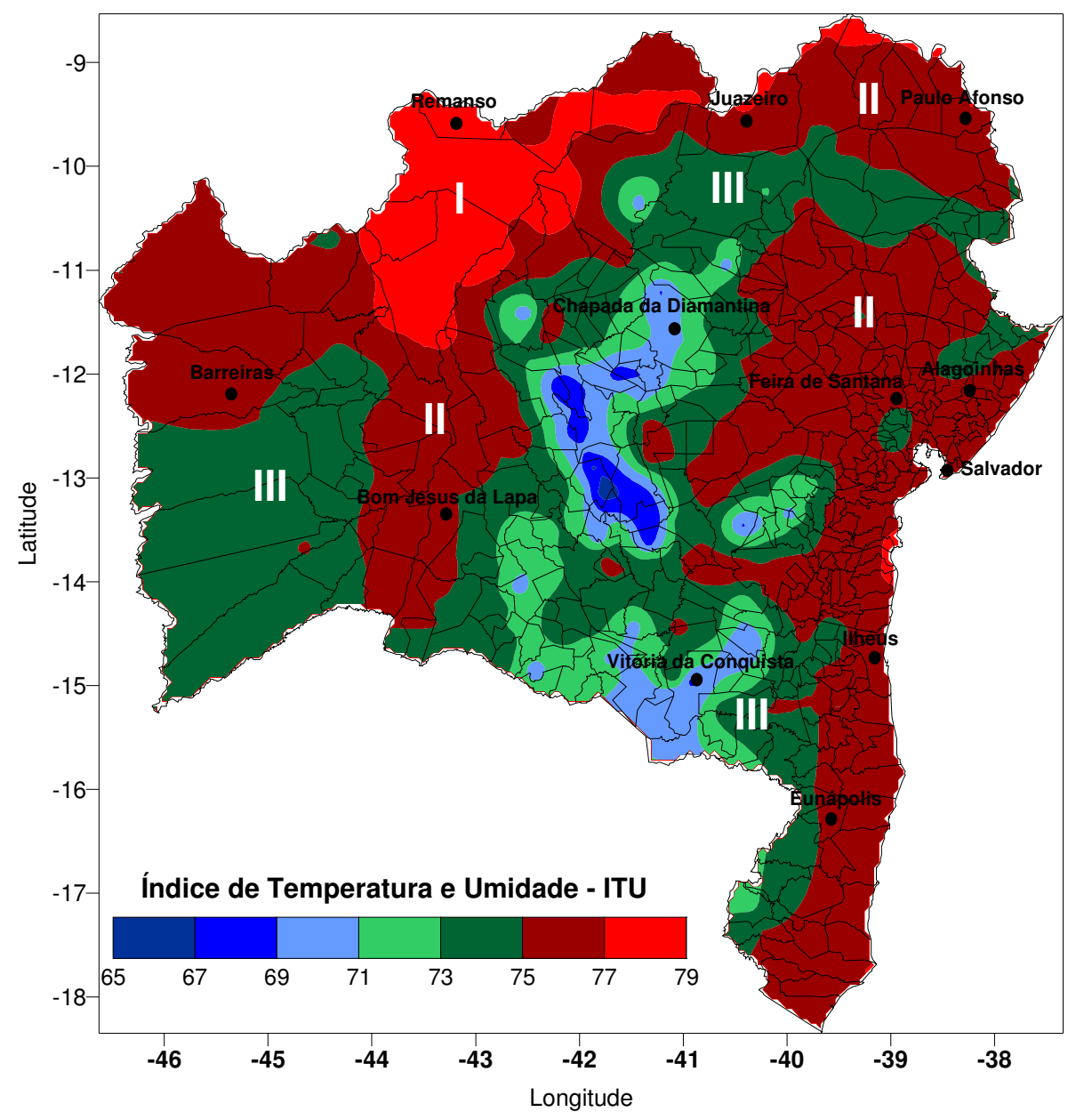

FIGURA 2. Zoneamento Bioclimático para bovinos, baseado no índice de temperatura e umidade (ITU) referente aos meses mais quentes do ano para a Bahia.

Na espacialização do declínio da produção, pode-se observar, pela Figura 3, que nas Zonas I e II, localizadas nas regiões norte e semi-árida da Bahia, poderão ocorrer declínios na produção de leite de 0 a $1 \mathrm{~kg}$ de leite por dia para vacas que produzem $10 \mathrm{~kg}$ nos meses mais quentes. Também no litoral baiano, há ocorrência de declínio na produção devido à alta umidade, mesmo em vacas com nível de produção baixo. Nas regiões da Chapada da Diamantina e de Vitória da Conquista, poderão ocorrer aumentos da produção de leite de um a dois litros por dia, estando de acordo com os dados de ITU que demonstram condições climáticas favoráveis dessas regiões, mesmo nos meses de verão. 


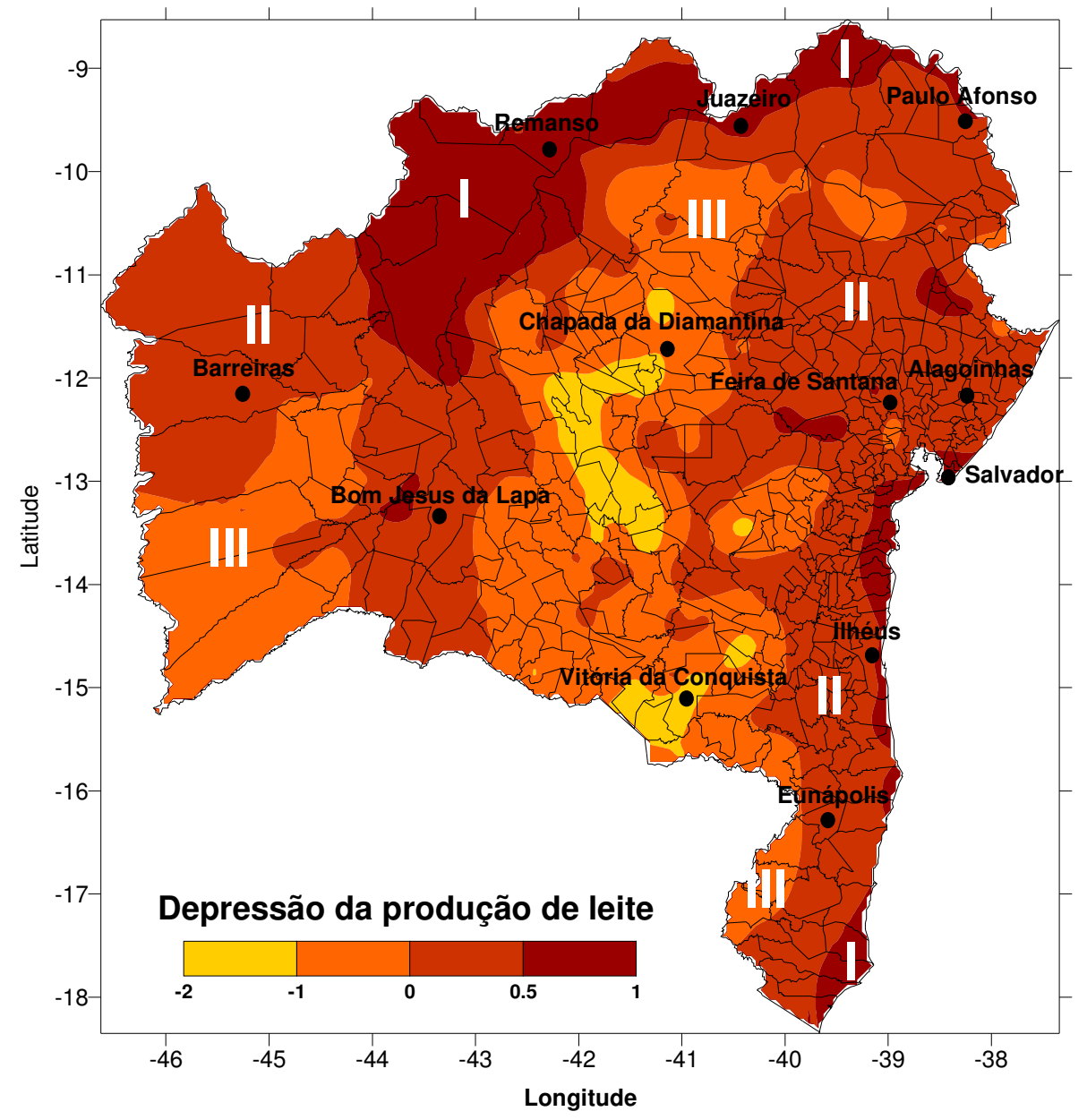

FIGURA 3. Espacialização da depressão da produção de leite (DPL) para vacas que produzem $10 \mathrm{~kg}$ na Bahia, para as condições ambientais de verão.

Na Figura 4, observa-se perda maior na produção de leite na Bahia devido o nível de produção das vacas ser maior. Essas vacas, com níveis de produção de $25 \mathrm{~kg}$ de leite por dia, necessitam de maior quantidade de alimentos, o que acarreta maior atividade metabólica, que produz grandes quantidades de calor, interferindo no seu desempenho leiteiro. Na Zona Bioclimática I, localizada no norte da Bahia e dentro da área semi-árida, pode-se observar perda diária de 3 a 4,5 $\mathrm{kg}$ de leite por dia. $\mathrm{Na} Z$ Zona Bioclimática II, localizada no norte e no litoral da Bahia, verificam-se possibilidades de perdas de 2 a $3 \mathrm{~kg}$ de leite por dia. Na Zona Bioclimática III, localizada principalmente na região oeste da Bahia, observa-se perda de leite de 1 a $2 \mathrm{~kg}$ por dia. As regiões Central (Chapada da Diamantina) e Centro-Sul da Bahia (Vitória da Conquista), mesmo nos meses quentes, apresentam ambiente mais favorável à produção de leite, com ITU menores, melhores para animais com maior nível de produção. Esses dados estão de acordo com JOHNSON (1980), que verificou que, para valores de ITU acima de 72, à medida que o ITU se elevou, a produção de leite foi declinando, sendo o declínio mais acentuado nas vacas de mais alta produção. JOHNSON (1980) observou que vacas de baixa produção $\left(13 \mathrm{~kg} \mathrm{dia}^{-1}\right)$ foram menos afetadas com ITU de 76 do que as de alta produção $\left(22 \mathrm{~kg} \mathrm{dia}^{-1}\right)$. 


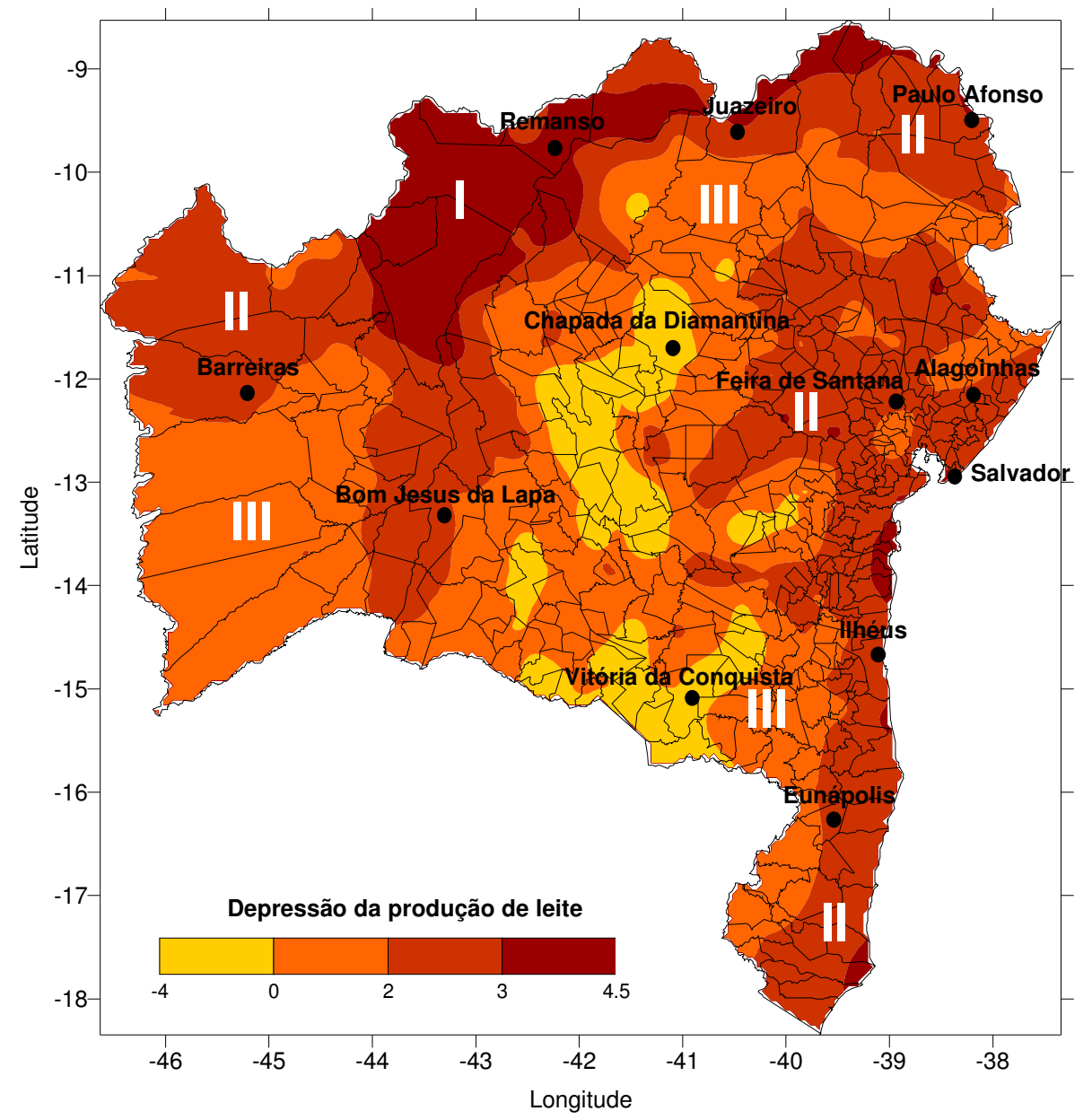

FIGURA 4. Espacialização da depressão da produção de leite (DPL) para vacas que produzem $25 \mathrm{~kg}$ na Bahia, para as condições ambientais de verão.

A manutenção, ou mesmo o aumento da produção, pode ser conseguida por meio de técnicas de manejo relacionadas com o condicionamento do ambiente animal. Com o objetivo de interferir no ambiente natural e impedir o estresse calórico dos animais, vários artifícios podem ser utilizados, como o sombreamento, que pode reduzir cerca de $30 \%$ ou mais na carga térmica da radiação solar (CTR), quando comparada à carga recebida pelo animal ao ar livre (BAÊTA \& SOUZA, 1997); também poderá ser utilizado o resfriamento do próprio ar ambiente e diretamente do animal, por meio de ventiladores, e indiretamente pelo resfriamento dos elementos construtivos, como as coberturas.

\section{CONCLUSÕES}

Com o zoneamento bioclimático e a espacialização do declínio na produção de leite para a Bahia, podem-se conhecer as regiões de desconforto térmico para o gado leiteiro e as suas possíveis perdas devido às condições climáticas desfavoráveis, sendo essas ferramentas importantes para tomadas de decisão de técnicos e produtores que explorarão animais de maior nível de produção e de maior sensibilidade para as condições climáticas.

\section{REFERÊNCIAS}

ARCARO JÚNIOR, I.; ARCARO, J.R.P.; POZZI, C.R.; MATARAZZO, V.; FAGUNDES, H. Produção e comosição de leite de vacas holandesas em sala de espera climatizada. In: CONGRESSO 
BRASILEIRO DE BIOMETEOROLOGIA, 3., 2001, Maringá. Anais... Maringá: Sociedade Brasileira de Biometeorologia, 2001. 1 CD-ROM.

BACCARI JÚNIOR, F. Manejo ambiental para produção de leite em climas quentes. In: CONGRESSO BRASILEIRO DE BIOCLIMATOLOGIA, 2., 1998, Goiania. Anais... Goiânia: Sociedade Brasileira de Biometeorologia, 1998. p.136-61.

BAÊTA, F.C.; SOUZA, C.F. Ambiência em edificações rurais - conforto animal. Viçosa: Editora UFV, 1997. 246 p.

BUFFINGTON, D.E.; COLLIER, R.J.; CANTON, G.H. Shede managemente systems to reduce heat stress for dairy cows. St. Joseph: American Society of Agricultural Engineers, 1982. 16 p.(Paper 824061).

CAMPOS, A.T.; PIRES, M.F.A.; CAMPOS, A.T.; STOCK, L.A.; CAMPOS, D.S.; RESENDE, J.C.; XAVIER, D.F. Efeito do estresse calórico sobre a produção de leite de vacas Holandesas na Região de Coronel Pacheco - MG. In: REUNIÃO DA SOCIEDADE BRASILEIRA DE ZOOTECNIA, 30., 2002, Recife. Anais... Recife: Sociedade Brasileira de Zootecnia, 2002. 1 CD-ROM.

CHEN, K.H.; HUBER, J.T.; SIMAS, J.; THEURER, C.B.; ARMSTRONG, D.V.; WANDERLEY, R.C.; SIMAS, J.M.; CHAN, S.C.; SULLIVAN, J.L. Effect of protein quality and evaporative cooling on lactational performance of Holstein cows in hot weather. Journal of Dairy Science, Champaign, v.76, n.3, p.816-25, 1993.

COLLIER, R.J.; ELEY, R.M.; SHARMA, A.K.; PEREIRA, R.M.; BUFFINGTON, D.E. Shade management in subtropical environment for milk yield and composition in Holstein an Jersey cows. Journal of Dairy Science, Champaign, v.64, p.844-9, 1981.

COLLIER, R.J.; BEEDE, D.K. Thermal stress as a factor associated with nutrient requirements and interrelationships. In: McDOWELL, L.R. (Ed.). Animal feeeding and nutrition. Orlando: Academic Press, 1985. p.59-71. (Series of monographs)

HAHN, G. Bioclimatologia e instalações zootécnicas. Jaboticabal: FUNEP, 1993. 28 p.

IGONO, M.O.; BJTVEDT, G.; SANFORD-CRANE, H.T. Environmental profile and critical temperature effects on milk prodution of Holsteins cows in desert climate. International Journal of Biometeorology, Heidelberg, v.36, p.77-87, 1992.

JOHNSON, H. Environmental management of cattle to minimize the stress of climatic change. International Journal of Biometeorology, Heidelberg, v.24, n.1, p.65-78, 1980.

McDOWELL, R.E.; JOHNSTON, J.E. Research under field conditions. In: NATIONAL ACADEMY OF SCIENCES. A guide to environmental research on animals. Washington, 1971. p.306-59.

McDOWELL, R.E.; HOOVEN, N.M.; CAMOENS, J.K. Effect of climate on performance of Holstein in first lactation. Journal of Dairy Science, Champaign, v.59, n.5, p.965-73, 1976.

ROSENBERG, L.J.; BIAD, B.L.; VERNS, S.B. Human and animal biometeorology. In: MICROCLIMATE, the biological environment. New York: Wiley-interscience Publication, 1983. $485 \mathrm{p}$.

TEIXEIRA, A.H.C.; SOUZA, R.A.; RIBEIRO, P.H.B.; COSTA, W.P.L.B. Espacialização da umidade relativa do ar no Estado da Bahia. In: CONGRESSO BRASILEIRO DE AGROMETEOROLOGIA, 12., 1973, Fortaleza. Anais... Fortaleza: Sociedade Brasileira de Agrometereologia, 2001. p.73-4.

THON, E.C. Cooling degress-day air conditioning, heating, and ventilating. Transactions of the $A S H R A E$, St. Joseph, v.55, n.7, p.65-72, 1958. 